\title{
Inverse Spectral Theory for a Singular Sturm Liouville Operator with Coulomb Potential
}

\author{
Etibar S. Panakhov1, Ismail Ulusoy ${ }^{2}$ \\ ${ }^{1}$ Department of Mathematics, Firat University, Elazig, Turkey \\ ${ }^{2}$ Department of Mathematics, Adiyaman University, Adiyaman, Turkey \\ Email: epenahov@firat.edu.tr, iulusoy@adiyaman.edu.tr
}

Received 21 September 2015; accepted 18 January 2016; published 21 January 2016

Copyright (C) 2016 by authors and Scientific Research Publishing Inc.

This work is licensed under the Creative Commons Attribution International License (CC BY). http://creativecommons.org/licenses/by/4.0/

c) (i) Open Access

\section{Abstract \\ We consider the inverse spectral problem for a singular Sturm-Liouville operator with Coulomb potential. In this paper, we give an asymptotic formula and some properties for this problem by using methods of Trubowitz and Poschel.}

\section{Keywords}

\section{Coulomb Potential, Asymptotic Formula, Normalizing Eigenfunction}

\section{Introduction}

The Sturm-Liouville equation is a second order linear ordinary differential equation of the form

$$
-\frac{\mathrm{d}}{\mathrm{d} x}\left(p(x) \frac{\mathrm{d} y}{\mathrm{~d} x}\right)+(l(x)-\lambda r(x)) y(x)=0
$$

for some $\lambda \in \mathbb{C}, x \in I=[a, b]$, and $y \in C^{2}(I)$. It was first introduced in an 1837 publication [1] by the eminent French mathematicians Joseph Liouville and Jacques Charles François Sturm. The Sturm-Liouville Equation (1.1) can easily be reduced to form

$$
-y^{\prime \prime}+q(x) y=\lambda y
$$

If we assume that $p(x)$ has a continuous first derivative, and $p(x), r(x)$ have a continuous second derivative, then by means of the substitutions 


$$
z=\frac{1}{c} \int_{a}^{x}\left(\frac{r(x)}{p(x)}\right)^{1 / 2} \mathrm{~d} x, \quad u=(r(x) p(x))^{1 / 4} y, \quad \mu=c \lambda,
$$

where $c$ is given by

$$
c=\frac{1}{\pi} \int_{a}^{b}\left(\frac{r(x)}{p(x)}\right)^{1 / 2} \mathrm{~d} x,
$$

Equation (1.1) assumes the form (1.2) replaced by $\mu$; where

$$
q(z)=\frac{\phi^{\prime \prime}(z)}{\phi(z)}-c^{2} \frac{l(x)}{r(x)}, \quad \phi(z)=(r(x) p(x))^{1 / 4} .
$$

The transformation of the general second order equation to canonical form and the asymptotic formulas for the eigenvalues and eigenfunctions was given by Liouville. A deep study of the distribution of the zeros of eigenfunctions was done by Sturm. Firstly, the formula for the distribution of the eigenvalues of the single dimensional Sturm operator defined in the whole of the straight-line axis with increasing potential in the infinity was given by Titchmarsh in 1946 [2] [3]. Titchmarsh also showed the distribution formula for the Schrödinger Operator. In later years, Levitan improved the Titchmarsh's method and found important asymptotic formula for the eigenvalues of different differential operators [4] [5]. Sturm-Liouville problems with a singularity at zero have various versions. The best known case is the one studied by Amirov [6] [7], in which the potential has a Coulomb-type singularity

$$
-y^{\prime \prime}+\frac{1}{x} y+q(x) y=\lambda y
$$

at the origin. In these works, properties of spectral characteristic were studied for Sturm-Liouville operators with Coulomb potential, which have discontinuity conditions inside a finite interval. Panakhov and Sat estimated nodal points and nodal lengths for the Sturm-Liouville operators with Coulomb potential [8]-[10]. Basand Metin defined a fractional singular Sturm-Liouville operator having Coulomb potential of type $A / x$ [11].

Let's give some fundamental physical properties of the Sturm-Liouville operator with Coulomb potential. Learning about the motion of electrons moving under the Coulomb potential is of significance in quantum theory. Solving these types of problems provides us with finding energy levels of not only hydrogen atom but also single valance electron atoms such as sodium. For the Coulomb potential is given by $U=\frac{-e^{2}}{r}$, where $r$ is the radius of the nucleus, $e$ is electronic charge. According to this, we use time-dependent Schrödinger equation

$$
i h \frac{\partial \psi}{\partial t}=-\frac{h^{2}}{2 m} \frac{\partial^{2} \psi}{\partial x^{2}}+U(x, y, z) \psi, \quad \int_{R^{3}}|\psi|^{2} \mathrm{~d} x \mathrm{~d} y \mathrm{~d} z=1,
$$

where $\psi$ is the wave function, $h$ is Planck's constant and $m$ is the mass of electron.

In this equation, if the Fourier transform is applied

$$
\tilde{\psi}=\frac{1}{\sqrt{2 \pi}} \int_{-\infty}^{\infty} \mathrm{e}^{-i \lambda t} \psi \mathrm{d} t
$$

it will convert to energy equation dependent on the situation as follows:

$$
\frac{h^{2}}{2 m} \nabla^{2} \tilde{\psi}+\tilde{U} \tilde{\psi}=E \tilde{\psi} .
$$

Therefore, energy equation in the field with Coulomb potential becomes

$$
-\frac{h^{2}}{2 m} \nabla^{2} \tilde{\psi}+\left(E+\frac{e^{2}}{r}\right) \tilde{\psi}=0 .
$$

If this hydrogen atom is substituted to other potential area, then energy equation becomes 


$$
-\frac{h^{2}}{2 m} \nabla^{2} \tilde{\psi}+\left(E+\frac{e^{2}}{r}+q(x, y, z)\right) \tilde{\psi}=0 .
$$

If we make the necessary transformation, then we can get a Sturm-Liouville equation with Coulomb potential

$$
-y^{\prime \prime}+\left[\frac{1}{x}+q(x)\right] y=\lambda y
$$

where $\lambda$ is a parameter which corresponds to the energy [12].

Our aim here is to find asymptotic formulas for singular Sturm-Liouville operatör with Coulomb potential with domain

$$
\left\{y \in L^{2}[0,1]: y, y^{\prime} \text { are absolutely continuous on }(0,1], L y \in L^{2}[0,1] \text { and } y(1)=0\right\} \sim
$$

$\square$ Also, we give the normalizing eigenfunctions and spectral functions.

\section{Basic Properties}

We consider the singular Sturm-Liouville problem

$$
-y^{\prime \prime}+\left[\frac{1}{x} y+q(x)\right] y=\lambda y, \lambda \in C
$$

where the function $q(x) \in L^{2}[0,1]$. Let us denote by $\varphi(x, \lambda, q)$ the solution of (2.1) satisfying the initial condition

$$
\varphi(0, \lambda, q)=0, \quad \varphi^{\prime}(0, \lambda, q)=1
$$

and by $\psi(x, \lambda, q)$ the solution of same equation, satisfying the initial condition

$$
\psi(0, \lambda, q)=1, \quad \psi^{\prime}(0, \lambda, q)=0
$$

Lemma 1. The solution of problem (2.1) and (2.2) has the following form:

$$
\varphi(x, \lambda, q)=\frac{\sin \sqrt{\lambda} x}{\sqrt{\lambda}}+\int_{0}^{x} \frac{\sin \sqrt{\lambda}(x-t)}{\sqrt{\lambda}}\left(\frac{1}{t}+q(t)\right) \varphi(t, \lambda, q) \mathrm{d} t
$$

where $q(x) \in L^{2}[0,1]$.

Proof. Since $\varphi(x, \lambda, q)$ satisfies Equation (2.1), we have

$$
\begin{aligned}
& \int_{0}^{x} \frac{\sin \sqrt{\lambda}(x-t)}{\sqrt{\lambda}}\left(\frac{1}{t}+q(t)\right) \varphi(t, \lambda, q) \mathrm{d} t \\
& =\int_{0}^{x} \frac{\sin \sqrt{\lambda}(x-t)}{\sqrt{\lambda}} \varphi^{\prime \prime}(t, \lambda, q) \mathrm{d} t+\lambda \int_{0}^{x} \sin \sqrt{\lambda}(x-t) \varphi(t, \lambda, q) \mathrm{d} t
\end{aligned}
$$

Integrating the first integral on the right side by parts twice and taking the conditions (2.2) into account, we find that

$$
\int_{0}^{x} \frac{\sin \sqrt{\lambda}(x-t)}{\sqrt{\lambda}}\left(\frac{1}{t}+q(t)\right) \varphi(t, \lambda, q) \mathrm{d} t=\varphi(x, \lambda, q)-\sin \sqrt{\lambda} x
$$

which is (2.4).

Lemma 2. The solution of problem (2.1) and (2.3) has the following form:

$$
\psi(x, \lambda, q)=\cos \sqrt{\lambda} x+\int_{0}^{x} \frac{\sin \sqrt{\lambda}(x-t)}{\sqrt{\lambda}}\left(\frac{1}{t}+q(t)\right) \psi(t, \lambda, q) \mathrm{d} t
$$

Proof. The proof is the same as that of Lemma 1.

Now we give some estimates of $\varphi$ and $\psi$ which will be used later. For each fixed $x$ in $[0,1]$ the map $(\lambda, q) \rightarrow \varphi(x, \lambda, q)$ is an entire function on $C \times L^{2}[0,1]$ which is real-valued on $R \times L_{R}^{2} \quad$ [13]. Using the esti- 
mate

$$
|\sin z| \leq \frac{c z}{1+|z|} \mathrm{e}^{\operatorname{Im}|z|}
$$

we get

$$
|\varphi(x, \lambda, q)| \leq \frac{c x}{1+|\sqrt{\lambda} x|} \mathrm{e}^{\operatorname{Im}|\sqrt{\lambda} x|}+\int_{0}^{x} \frac{c(x-t)}{1+|\sqrt{\lambda}(x-t)|} \mathrm{e}^{\operatorname{Im}|\sqrt{\lambda} x|}\left|\frac{1}{t}+q(t)\right| \frac{c t}{1+|\sqrt{\lambda} t|} \mathrm{e}^{\operatorname{Im}|\sqrt{\lambda} t|} \mathrm{d} t .
$$

Since $0 \leq t \leq x \leq 1$ and

$$
\frac{x-t}{1+|\sqrt{\lambda}|(x-t)} \leq \frac{x}{1+|\sqrt{\lambda}| x}
$$

we have

$$
|\varphi(x, \lambda, q)| \leq \frac{c x}{1+|\sqrt{\lambda} x|} \mathrm{e}^{\operatorname{Im}|\sqrt{\lambda} x|}
$$

From (2.6) the inequality is easily checked

$$
\left|\varphi(x, \lambda, q)-\frac{\sin \sqrt{\lambda} x}{\sqrt{\lambda}}\right| \leq \frac{c x}{1+|\sqrt{\lambda} x|} \mathrm{e}^{\operatorname{Im}|\sqrt{\lambda} x|}\left[\int_{0}^{x} \frac{1+t q(t)}{1+|\sqrt{\lambda} t|} \mathrm{d} t\right]
$$

where $c$ is uniform with respect to $q$ on bounded sets in $L^{2}[0,1]$.

Lemma 3 (Counting Lemma). [13] Let $q \in L^{2}[0,1]$ and $N>\mathrm{e}^{2\|q\|}$ be an integer. Then $\varphi(1, \lambda, q)$ has exactly $N$ roots, counted with multiplicities, in the open half plane

$$
\operatorname{Re} \lambda<(N+1 / 2)^{2} \pi^{2}
$$

and for each $n>N$, exactly one simple root in the egg shaped region

$$
|\lambda-n \pi|<\pi / 2
$$

There are no other roots.

From this Lemma there exists an integer $N$ such that for every $n>N$ there is only one $\mu_{n}(q)$ eigenvalue in $\left\{\lambda \in \mathbb{R},\left|\lambda^{1 / 2}-(n+1 / 2) \pi\right|<\pi / 2\right\}$. Thus for every $n \geq N$

$$
\mu_{n}(q)^{1 / 2}=\left(n+\frac{1}{2}\right) \pi+\varepsilon_{n}, \quad\left|\varepsilon_{n}\right|<\frac{\pi}{2}, \quad n \geq N
$$

$N$ can be chosen independent of $q$ on bounded sets of $L_{R}^{2}[0,1]$. Following theorem [13] shows that the eigenvalues $\left\{\mu_{n}(q)\right\}_{n \geq 1}$ are the zeroes of the map $\lambda \rightarrow \varphi(1, \lambda, q)$ and these zeroes are simple.

Theorem 1. If $\lambda$ is Dirichlet eigenvalue of $q$ in $L^{2}$, then

$$
\frac{\partial \varphi}{\partial \lambda}(1, \lambda, q) \frac{\partial \varphi}{\partial x}(1, \lambda, q)=\int_{0}^{1}(\varphi(t, \lambda, q))^{2} \mathrm{~d} t=\|\varphi(t, \lambda, q)\|^{2}>0 .
$$

In particular, $\frac{\partial \varphi}{\partial \lambda}(1, \lambda, q) \neq 0$. Thus, all roots of $\varphi(1, \lambda, q)$ are simple.

Proof. The proof is similar as that of ([13], Pöschel and Trubowitz).

\section{Asymptotic Formula}

We need the following lemma for proving the main result.

Lemma 4. For every $f$ in $L^{2}[0,1]$, 


$$
\int_{0}^{1} \frac{\cos \sqrt{\mu_{n}(q)} \sin ^{2} \sqrt{\mu_{n}(q)} t}{\mu_{n}(q)} f(t) \mathrm{d} t=\frac{\cos \sqrt{\mu_{n}(q)}}{\mu_{n}(q)} \int_{0}^{1} \frac{f(t)}{2} \mathrm{~d} t+O\left(\frac{1}{n^{3}}\right)
$$

and

$$
\int_{0}^{1} \frac{\sin \sqrt{\mu_{n}(q)} \sin 2 \sqrt{\mu_{n}(q)} t}{2 \mu_{n}(q)} f(t) \mathrm{d} t=O\left(\frac{1}{n^{3}}\right) .
$$

Proof. Firstly, we shall prove the relation (3.1)

$$
\int_{0}^{1} \frac{\cos \sqrt{\mu_{n}(q)} \sin ^{2} \sqrt{\mu_{n}(q)} t}{\mu_{n}(q)} f(t) \mathrm{d} t
$$

By the Cauchy-Schwarz inequality, we get

$$
\int_{0}^{1} \frac{\cos \sqrt{\mu_{n}(q)} \sin ^{2} \sqrt{\mu_{n}(q)} t}{\mu_{n}(q)} f(t) \mathrm{d} t \leq \frac{\cos \sqrt{\mu_{n}(q)}}{\mu_{n}(q)}\left(\int_{0}^{1} \frac{f(t)}{2} \mathrm{~d} t-\int_{0}^{1} \frac{\cos ^{2} 2 \sqrt{\mu_{n}(q)} t}{4} \mathrm{~d} t \int_{0}^{1} f^{2}(t) \mathrm{d} t\right) .
$$

Since $f$ is in $L^{2}[0,1]$, the last two integrals are equal to

$$
\int_{0}^{1} \frac{\cos ^{2} 2 \sqrt{\mu_{n}(q)} t}{4} \mathrm{~d} t \int_{0}^{1} f^{2}(t) \mathrm{d} t=O\left(\frac{1}{n}\right)
$$

So (3.3) is equivalent to

$$
\int_{0}^{1} \frac{\cos \sqrt{\mu_{n}(q)} \sin ^{2} \sqrt{\mu_{n}(q)} t}{\mu_{n}(q)} f(t) \mathrm{d} t=\frac{\cos \sqrt{\mu_{n}(q)}}{\mu_{n}(q)}\left(\int_{0}^{1} \frac{f(t)}{2} \mathrm{~d} t+O\left(\frac{1}{n}\right)\right) .
$$

Finally, we shall prove the relation (3.2)

$$
\begin{aligned}
& \int_{0}^{1} \frac{\sin \sqrt{\mu_{n}(q)} \sin 2 \sqrt{\mu_{n}(q)} t}{2 \mu_{n}(q)} f(t) \mathrm{d} t \\
& \leq \frac{\sin \sqrt{\mu_{n}(q)}}{2 \mu_{n}(q)} \int_{0}^{1} \sin ^{2} 2 \sqrt{\mu_{n}(q)} t \int_{0}^{1} f^{2}(t) \mathrm{d} t=O\left(\frac{1}{n^{3}}\right) .
\end{aligned}
$$

This proves the lemma.

The main result of this article is the following theorem:

Theorem 2. For $q(x) \in L^{2}[0,1]$,

$$
\mu_{n}(q)=\left(n+\frac{1}{2}\right)^{2} \pi^{2}\left[1+\frac{2}{\int_{0}^{1} q(t) \mathrm{d} t}\right]^{2}+O\left(\frac{1}{n^{4}}\right) .
$$

Proof of the Main Theorem. Since $\varphi(0, \lambda, q)=0$, it must be $\varphi(1, \lambda, q)=0$. Because $\varphi(x, \lambda, q)$ is a nontrivial solution of Equation (2.1) satisfying Dirichlet boundary conditions, we have

$$
0=\varphi\left(1, \mu_{n}(q), q\right)=\frac{\sin \sqrt{\mu_{n}(q)}}{\sqrt{\mu_{n}(q)}}+\int_{0}^{1} \frac{\sin \sqrt{\mu_{n}(q)}(1-t)}{\sqrt{\mu_{n}(q)}}\left(\frac{1}{t}+q(t)\right) \varphi\left(t, \mu_{n}(q), q\right) \mathrm{d} t
$$

From (2.7) someone gets the inequality

$$
\varphi\left(t, \mu_{n}(q), q\right) \leq \frac{\sin \sqrt{\mu_{n}(q)} t}{\sqrt{\mu_{n}(q)}}+\frac{c t}{1+\left|\sqrt{\mu_{n}(q)}\right| t} \mathrm{e}^{\operatorname{Im} \sqrt{\mu_{n}(q)} \mid t} \int_{0}^{1} \frac{1+|q(t)| t}{1+\left|\sqrt{\mu_{n}(q)}\right| t} \mathrm{~d} t
$$

From (3.5) integral in the equation of (3.4) takes the form 


$$
\begin{aligned}
& \int_{0}^{1} \frac{\sin \sqrt{\mu_{n}(q)}(1-t)}{\sqrt{\mu_{n}(q)}}\left(\frac{1}{t}+q(t)\right) \varphi\left(t, \mu_{n}(q), q\right) \mathrm{d} t \\
& \leq \int_{0}^{1} \frac{\sin \sqrt{\mu_{n}(q)}(1-t)}{\sqrt{\mu_{n}(q)}}\left(\frac{1}{t}+q(t)\right) \frac{\sin \sqrt{\mu_{n}(q)} t}{\sqrt{\mu_{n}(q)}} \mathrm{d} t \\
& \quad+\int_{0}^{1} \frac{\sin \sqrt{\mu_{n}(q)}(1-t)}{\sqrt{\mu_{n}(q)}}\left(\frac{1}{t}+q(t)\right) \frac{c t}{1+\left|\sqrt{\mu_{n}(q)}\right| t} \mathrm{e}^{\left|\operatorname{lm} \sqrt{\mu_{n}(q)}\right|} \int_{0}^{1} \frac{1+|q(s)| s}{1+\left|\sqrt{\mu_{n}(q)}\right| s} \mathrm{~d} s \mathrm{~d} t
\end{aligned}
$$

By using difference formulas for sine we have

$$
\begin{aligned}
& \int_{0}^{1} \frac{\sin \sqrt{\mu_{n}(q)}(1-t)}{\sqrt{\mu_{n}(q)}}\left(\frac{1}{t}+q(t)\right) \varphi\left(t, \mu_{n}(q), q\right) \mathrm{d} t \\
& \leq \int_{0}^{1} \frac{\left(\sin \sqrt{\mu_{n}(q)} \sin 2 \sqrt{\mu_{n}(q)} t\right) / 2-\sin ^{2} \sqrt{\mu_{n}(q)} t \cos \sqrt{\mu_{n}(q)}}{t \mu_{n}(q)} \mathrm{d} t \\
& +\int_{0}^{1} \frac{\left.\sin \sqrt{\mu_{n}(q)} \sin 2 \sqrt{\mu_{n}(q)} t\right) / 2-\sin ^{2} \sqrt{\mu_{n}(q)} t \cos \sqrt{\mu_{n}(q)}}{\mu_{n}(q)} q(t) \mathrm{d} t+O\left(\frac{1}{n^{3}}\right)
\end{aligned}
$$

From Lemma 4 we get

$$
\int_{0}^{1} \frac{\sin \sqrt{\mu_{n}(q)}(1-t)}{\sqrt{\mu_{n}(q)}}\left(\frac{1}{t}+q(t)\right) \varphi\left(t, \mu_{n}(q), q\right) \mathrm{d} t \leq-\frac{\cos \sqrt{\mu_{n}(q)}}{\mu_{n}(q)} \int_{0}^{1} \frac{q(t)}{2} \mathrm{~d} t+O\left(\frac{1}{n^{3}}\right)
$$

Thus, by using this inequality (3.4) can be written in the form

$$
0=\frac{\sin \sqrt{\mu_{n}(q)}}{\sqrt{\mu_{n}(q)}}-\frac{\cos \sqrt{\mu_{n}(q)}}{\mu_{n}(q)} \int_{0}^{1} \frac{q(t)}{2} \mathrm{~d} t+O\left(\frac{1}{n^{3}}\right)
$$

From (2.8) we conclude that

$$
0=\sin \left[\left(n+\frac{1}{2}\right) \pi+\varepsilon_{n}\right]-\frac{\cos \left[\left(n+\frac{1}{2}\right) \pi+\varepsilon_{n}\right]}{\left[\left(n+\frac{1}{2}\right) \pi+\varepsilon_{n}\right]} \frac{1}{2} \int_{0}^{1} q(t) \mathrm{d} t+O\left(\frac{1}{n^{3}}\right)
$$

Since $\sin \left[\left(n+\frac{1}{2}\right) \pi+\varepsilon_{n}\right]=(-1)^{n} \cos \varepsilon_{n}$ and $\cos \left[\left(n+\frac{1}{2}\right) \pi+\varepsilon_{n}\right]=-(-1)^{n} \sin \varepsilon_{n}$, (3.7) is equivalent to

$$
\tan \varepsilon_{n}=\frac{2\left(n+\frac{1}{2}\right) \pi}{\int_{0}^{1} q(t) \mathrm{d} t}+O\left(\frac{1}{n}\right)
$$

So we get

$$
\varepsilon_{n}=\frac{2\left(n+\frac{1}{2}\right) \pi}{\int_{0}^{1} q(t) \mathrm{d} t}+O\left(\frac{1}{n}\right)
$$

From (2.8) we have 


$$
\begin{aligned}
\mu_{n}(q) & =\left[\left(n+\frac{1}{2}\right) \pi+\frac{2\left(n+\frac{1}{2}\right) \pi}{\int_{0}^{1} q(t) \mathrm{d} t}+O\left(\frac{1}{n}\right)\right]^{2} \\
& =\left(n+\frac{1}{2}\right)^{2} \pi^{2}\left[1+\frac{2}{\int_{0}^{1} q(t) \mathrm{d} t}\right]^{2}+O\left(\frac{1}{n^{4}}\right)
\end{aligned}
$$

In this case, the theorem is proved.

From this theorem, the map

$$
q \rightarrow \mu(q)=\left(\mu_{1}(q), \mu_{2}(q), \cdots\right)
$$

from $q$ to its sequences of Dirichlet eigenvalues sends $L^{2}$ into $S$. Later, we need this map to characterize spectra which is equivalent to determining the image of $\mu$.

\section{Inverse Spectral Theory}

To each eigenvalue we associate a unique eigenfunction $g_{n}(x, q)$ normalized by

$$
\left\|g_{n}(x, q)\right\|=1
$$

Let's define the normalizing eigenfunction $g_{n}(x, q)$ :

$$
g_{n}(x)=\frac{\varphi\left(x, \mu_{n}, q\right)}{\left\|\varphi\left(x, \mu_{n}, q\right)\right\|}
$$

Lemma 5. For $q(x) \in L^{2}[0,1]$,

$$
g_{n}^{2}(x, q)=2 \sin ^{2} \sqrt{\mu_{n}} x+O\left(\frac{1}{n}\right)
$$

This estimate holds uniformly on bounded subsets of $[0,1] \times L^{2}$.

Proof. Let $\varphi_{n}(x)=\varphi\left(x, \mu_{n}(q), q\right)$ and $\psi_{n}(x)=\psi\left(x, \mu_{n}(q), q\right)$. By the basic estimate for $\varphi\left(x, \mu_{n}(q), q\right)$,

$$
\varphi\left(x, \mu_{n}(q), q\right)=\frac{\sin \sqrt{\mu_{n}(q)} x}{\sqrt{\mu_{n}(q)}}+O\left(\frac{1}{n^{2}}\right) .
$$

By using this estimate we have

$$
\varphi_{n}^{2}(x)=\frac{\sin ^{2} \sqrt{\mu_{n}} x}{\mu_{n}}+O\left(\frac{1}{n^{3}}\right)
$$

So we get

$$
\int_{0}^{1} \frac{\sin ^{2} \sqrt{\mu_{n}} x}{\mu_{n}} \mathrm{~d} x=\int_{0}^{1} \frac{1-\cos 2 \sqrt{\mu_{n}} x}{2 \mu_{n}} \mathrm{~d} x=\frac{1}{2 \mu_{n}}\left(1+O\left(\frac{1}{n}\right)\right) .
$$

Thus we conclude that

$$
\left\|\varphi_{n}(x)\right\|^{2}=\frac{1}{2 \mu_{n}}\left(1+O\left(\frac{1}{n}\right)\right)
$$

Dividing $\varphi_{n}(x)$ by $\left\|\varphi_{n}(x)\right\|^{2}$ we get

$$
g_{n}^{2}(x, q)=2 \sin ^{2} \sqrt{\mu_{n}} x+O\left(\frac{1}{n}\right) .
$$

Also, we need to have asymptotic estimates of the squares of the eigenfunctions and products 


$$
a_{n}(x, q)=\varphi_{n}(x) \psi_{n}(x), \quad n \geq 1
$$

Lemma 6. For $q(x) \in L^{2}[0,1]$,

$$
a_{n}(x, q)=\frac{\sin 2 \sqrt{\mu_{n}(q)} x}{2 \sqrt{\mu_{n}(q)}}+O\left(\frac{1}{n^{2}}\right)
$$

This estimate holds uniformly on bounded subsets of $[0,1] \times L^{2}$.

Proof. We know that

$$
\varphi\left(x, \mu_{n}(q), q\right)=\frac{\sin \sqrt{\mu_{n}(q)} x}{\sqrt{\mu_{n}(q)}}+O\left(\frac{1}{n^{2}}\right)
$$

By the basic estimate for $\psi\left(x, \mu_{n}(q), q\right)$, we have

$$
\psi\left(x, \mu_{n}(q), q\right)=\cos \sqrt{\mu_{n}(q)}+O\left(\frac{1}{n^{2}}\right)
$$

Hence,

$$
a_{n}(x, q)=\left[\frac{\sin \sqrt{\mu_{n}(q)} x}{\sqrt{\mu_{n}(q)}}+O\left(\frac{1}{n^{2}}\right)\right]\left[\cos \sqrt{\mu_{n}(q)}+O\left(\frac{1}{n^{2}}\right)\right]=\frac{\sin 2 \sqrt{\mu_{n}(q)} x}{2 \sqrt{\mu_{n}(q)}}+O\left(\frac{1}{n^{2}}\right)
$$

Let

$$
\kappa_{n}(q)=\log (-1)^{n} \frac{\partial \varphi}{\partial x}\left(1, \mu_{n}, q\right)
$$

The map $q \rightarrow \kappa_{n}(q)$ is real analytic on $L_{R}^{2}[0,1]$. Now we give asymptotic behavior for $\kappa_{n}(q)$.

Theorem 3. Each $\kappa_{n}, n \geq 1$ is a compact, real analytic function on $L^{2}$ with

$$
\kappa_{n}(q)=\frac{1}{2 \sqrt{\mu_{n}(q)}}\left\langle\sin 2 \sqrt{\mu_{n}(q)} x, q(x)\right\rangle+O\left(\frac{1}{n^{2}}\right)
$$

Its gradient is

$$
\frac{\partial \kappa_{n}}{\partial q(t)}=\frac{\sin 2 \sqrt{\mu_{n}(q)} x}{2 \sqrt{\mu_{n}(q)}}+O\left(\frac{1}{n^{2}}\right)
$$

The error terms are uniform on bounded subsets of $[0,1] \times L^{2}$.

Proof. From [14] we have

$$
\begin{aligned}
\frac{\partial \kappa_{n}}{\partial((t)} & =a_{n}(t, q)-g_{n n}^{2}(t, q) \int_{0}^{1} a_{n}(x, q) \mathrm{d} x \\
& =\frac{\sin 2 \sqrt{\mu_{n}(q)} x}{2 \sqrt{\mu_{n}(q)}}-2 \sin ^{2} \sqrt{\mu_{n}(q)} x \int_{0}^{1} \frac{\sin 2 \sqrt{\mu_{n}(q)} x}{2 \sqrt{\mu_{n}(q)}} \mathrm{d} x+O\left(\frac{1}{n^{2}}\right)
\end{aligned}
$$

So we calculate the integral

$$
\int_{0}^{1} \frac{\sin 2 \sqrt{\mu_{n}(q)} x}{2 \sqrt{\mu_{n}(q)}} \mathrm{d} x=\frac{1-\cos 2 \sqrt{\mu_{n}(q)}}{4 \mu_{n}(q)}
$$

Finally, since $\kappa_{n}(0)=0$, we get 


$$
\begin{aligned}
\kappa_{n}(q) & =\int_{0}^{1} \frac{\mathrm{d}}{\mathrm{d} t} \kappa_{n}(t q) \mathrm{d} t=\int_{0}^{1}\left\langle\frac{\sin 2 \sqrt{\mu_{n}(q)} t}{2 \sqrt{\mu_{n}(q)}}+O\left(\frac{1}{n^{2}}\right), q(t)\right\rangle \mathrm{d} t \\
& =\int_{0}^{1} \int_{0}^{1} \frac{\sin 2 \sqrt{\mu_{n}(q)} s}{2 \sqrt{\mu_{n}(q)}} q(s) \mathrm{d} s \mathrm{~d} t+O\left(\frac{1}{n^{2}}\right)
\end{aligned}
$$

By the Cauchy-Schwarz inequality, we prove the theorem.

Let

$$
\kappa(q)=\left\{n \kappa_{n}(q)\right\}_{n \geq 1}
$$

Formula (4.3) shows that $\kappa(q)$ belongs to $l_{R}^{2}$. By Theorem 3, the map

$$
q \rightarrow \kappa(q)=\left(\kappa_{1}(q), \kappa_{2}(q), \cdots\right)
$$

from $q$ to its sequences of $\kappa$-values maps $L^{2}$ into the $l_{1}^{2}$. So we obtain a map

$$
q \rightarrow(\kappa \times \mu)(q)=(\kappa(q), \mu(q))
$$

from $L^{2}$ into the $l_{1}^{2} \times S$.

Theorem 4. [13] $\kappa \times \mu$ is one-to-one on $L^{2}$.

Let $d_{q}(\kappa \times \mu)$ be the Frechet derivative of the map $\kappa \times \mu$ at $q$.

Theorem 5. [14] $d_{q}(\kappa \times \mu)$ is an isomorphism from $L_{R}^{2}[0,1]$ onto $l_{R}^{2} \times S$.

\section{References}

[1] Sturm, C. and Liouville, J. (1837) Extrait d.un m emoire sur le d eveloppement des fonctions en series dont les di erents terms sont assujettis a satisfaire a une $\mathrm{m}$ eme equation di er entielle lin eaire, contenant un param etre variable. Journal de Math ematiques Pures et Appliqu ees. Journal de Mathématiques Pures et Appliquées, 2, 220-233.

[2] Birkhoff, G.D. (1908) Boundary Value and Expansion Problems of Ordinary Linear Differential Equations. Transactions of the American Mathematical Society, 9, 219-231. http://dx.doi.org/10.1090/S0002-9947-1908-1500810-1

[3] Titchmarsh, E.C. (1946) Eigenfunction Expansions Associated with Second-Order Differential Equations. Vol. 1, Clarendon Press, Oxford.

[4] Titchmarsh, E.C. (1958) Eigenfunction Expansions Associated with Second-Order Differential Equations. Vol. 2, Clarendon Press, Oxford.

[5] Levitan, B.M. (1978) On the Determination of the Sturm-Liouville Operator from One and Two Spectra. Mathematics of the USSR Izvestija, 12, 179-193. http://dx.doi.org/10.1070/IM1978v012n01ABEH001844

[6] Amirov, R.Kh. (1985) Inverse Problem for the Sturm-Liouville Equation with Coulomb Singularity Its Spectra. Kand. Dissertasiya, Baku.

[7] Topsakal, N. and Amirov, R. (2010) Inverse Problem for Sturm-Liouville Operators with Coulomb Potential Which Have Discontinuity Conditions inside an Interval. Mathematical Physics, Analysis and Geometry, 13, 29-46. http://dx.doi.org/10.1007/s11040-009-9066-y

[8] Sat, M. and Panakhov, E.S. (2012) Inverse Nodal Problem for Sturm-Liouville Operators with Coulomb Potential. International Journal of Pure and Applied Mathematics, 80, 173-180.

[9] Sat, M. and Panakhov, E.S. (2013) Reconstruction of Potential Function for Sturm-Liouville Operator with Coulomb Potential. Boundary Value Problems, 2013, Article 49.

[10] Sat, M. (2014) Half Inverse Problem for the Sturm-Liouville Operator with Coulomb Potential. Applied Mathematics and Information Sciences, 8, 501-504. http://dx.doi.org/10.12785/amis/080207

[11] Bas, E. and Metin, F. (2013) Fractional Singular Sturm-Liouville Operator for Coulomb Potential. Advances in Difference Equations, Article ID: 300. http://dx.doi.org/10.1186/1687-1847-2013-300

[12] Blohincev, D.I. (1949) Foundations of Quantum Mechanics. GITTL, Moscow.

[13] Poeschel, J. and Trubowitz, E. (1987) Inverse Spectral Theory. Academic Press, San Diego.

[14] Guillot, J.-C. and Ralston, J.V. (1988) Inverse Spectral Theory for a Singular Sturm-Liouville Operatör on [0,1]. Journal of Differential Equations, 76, 353-373. http://dx.doi.org/10.1016/0022-0396(88)90080-0 